\title{
EREBEA
}

Revista de Humanidades

y Ciencias Sociales

Núm. 3 (2013), pp. 407-425

ISSN: 0214-0691

\section{LA SITUACIÓN LABORAL DE LA MUJER EN LA HUELVA DE I930. UN CASO ESPECIAL: LA PROSTITUCIÓN}

\author{
Encarnación Gil Rivera \\ Inés Ma Salas Lorca \\ Universidad de Huelva
}

\section{RESUMEN}

El propósito de este artículo es efectuar un análisis del papel de la mujer en el mundo del trabajo (entendido éste como trabajo extradoméstico), en la Huelva de 1930. Pretendemos mostrar el porcentaje de mujeres que trabajaba fuera de casa y en qué sectores lo hacían, analizando algunas cuestiones relacionadas con el tema, como la educación, el grupo social o la capacidad económica. Asimismo, hemos querido detenernos en un grupo especial de trabajadoras: las prostitutas. Para todo ello hemos utilizado como primera fuente documental el Padrón Municipal.

Palabras Clave

trabajo, mujer, Huelva, 1930, prostitución.

Fecha de recepción: 2 de abril de 2013

Fecha de aceptación: 31 de julio de 2013

Abstract

The aim of this paper is to analyze the role of women in the world of work (understood as work outside the home) in Huelva in 1930. We intend to show the percentage of women who worked outside the home and in what sectors they did, analyzing issues related to the topic, such as education, social groups or economic standing. We have given specific emphasis to a special working group: prostitutes. To carry out this study, we have used the Municipal Census as our first documental source.

\footnotetext{
KEYWORDS

labor, women, Huelva, 1930, prostitution.
} 

Dada la relevancia del empleo femenino a la hora de definir el proceso de modernización de una zona y estudiar su contemporaneidad, hemos considerado necesario efectuar un análisis del papel de la mujer en el mundo del trabajo, entendido éste como trabajo extradoméstico, es decir, el remunerado, con el objetivo de acercarnos al grado de modernización de Huelva en 1930 y compararlo con la media española. Pretendemos conocer qué porcentaje de mujeres trabajaba fuera de casa y en qué sectores lo hacían, analizando algunas cuestiones relacionadas con el tema, como son la educación, el grupo social o la capacidad económica. Asimismo, hemos querido detenernos en un colectivo especial de trabajadoras: las prostitutas, sobre las que se ha elaborado un pequeño estudio. Para todo ello vamos a utilizar como primera fuente documental el Padrón Municipal, que aporta valiosos datos acerca de estas cuestiones, datos que no aparecen reflejados en otro tipo de documentación.

La elección del año 1930 se justifica en base a la existencia de un gran vacío historiográfico. La Dictadura del general Berenguer es uno de los periodos peor conocidos de la historia onubense, ya que tan solo disponemos del estudio de Miguel Ángel García Díaz. En cambio, contamos con diferentes trabajos referentes a épocas inmediatamente anteriores, como los de María Antonia Peña Guerrero ${ }^{2}$, Cristóbal García García 3 , Ana María Mojarro Bayo ${ }^{4}$ y las publicaciones del ya citado Miguel Ángel García Díaz ${ }^{5}$ que nos aportan numerosos datos acerca de la Huelva de aquella época.

1 M. A. García Díaz, "Huelva (1930-1931): Entre la Dictadura y la República”. En Huelva en su historia - 2 época, vol. 9, 2002, pp.119-144.

2 M.A. Peña Guerrero, El sistema caciquil en la provincia de Huelva. Clase política y partidos (1898-1923). Córdoba, Ayuntamiento de Córdoba, 1993.

-Clientelismo político y poderes periféricos durante la Restauración. Huelva. 1874-1923. Huelva, Universidad de Huelva, 1998.

—-El impacto del golpe de Estado de Primo de Rivera en la provincia de Huelva”. En Cuadernos del INICE, Salamanca, 1989, n²6 - 27, pp.35-40.

—.La dictadura de Primo de Rivera en Huelva. Las transformaciones políticas inmediatas". En Aestuaria, Huelva, Diputación de Huelva, 1992, pp. 13-22.

3 C. García García, Partidos y elecciones. 1933 en Huelva. Huelva, Universidad de Huelva, 1996.

-Modernización política y pervivencias caciquiles. Huelva. 1931-1936. Huelva, Ayuntamiento de Huelva, 2001.

4 A. M. Mojarro Bayo, El puerto de Huelva durante la dictadura de Primo de Rivera (19231930). Huelva, Autoridad Portuaria de Huelva, 2008.

5 M. A. García Díaz, Vieja y nueva política: Huelva bajo el régimen de Primo de Rivera (19231930). Huelva, Ayuntamiento de Huelva, 2005.

- "La política educativa de Primo de Rivera en Ayamonte: el proyecto de creación de escuelas en 1924. En IV Jornadas de Historia de Ayamonte, Ayamonte, 2000, pp.159-173. 
Por otra parte, este estudio se enmarca dentro de un proyecto que pretende abordar la situación laboral de las féminas onubenses en el periodo republicano y las primeras décadas del Franquismo, realizando una comparación entre ambas épocas. Este primer trabajo supone, por tanto, el punto de partida de una investigación que nos conducirá finalmente a dibujar el panorama laboral femenino de la primera mitad del siglo XX.

En cuanto al espacio geográfico elegido, Huelva capital, pensamos que era necesario el abordaje de dicho tema ya que estamos ante un terreno virgen para el investigador. Así, mientras que existen algunos estudios relativos a otras provincias andaluzas, como Sevilla ${ }^{6}$ o Málaga ${ }^{7}$, no existe ninguna obra de estas características relativa al ámbito onubense. Por otra parte, la curiosidad por conocer una faceta más de la historia de nuestra tierra ha determinado también la elección del marco espacial.

Con respecto a las fuentes manuscritas utilizadas, ya hemos comentado la base principal de nuestra investigación es el Padrón Municipal de Huelva del año $1930^{8}$. Los padrones municipales proporcionan una cantidad importante de información, que nos acerca a la vida cotidiana de la población. A través de esta fuente el historiador obtiene datos como la edad, sexo, estado civil, el nivel económico, el nivel educativo, lugar de procedencia etc., que permiten elaborar el panorama diario de los habitantes de una zona concreta. Hemos de señalar, por otro lado, las limitaciones que esta fuente presenta. Por ejemplo, las actividades laborales femeninas no siempre aparecen reflejadas en ella, y aunque a menudo las señoras trabajaban ayudando a sus esposos en las tareas agrícolas o en alguna tienda familiar, este tipo de ocupaciones en la mayoría de las ocasiones no se consignaban en el Padrón, señalándose el consabido "su casa" en la casilla destinada a la profesión.

Debido a la extensión de esta fuente, se ha realizado un muestreo aleatorio, formado por un diez por ciento de los hogares inscritos, trabajando sobre un total de 1785 mujeres. A medida que nuestra investigación avance pretendemos utilizar la totalidad de la misma.

Por otra parte, el Censo ${ }^{9}$ y el Anuario Estadístico de $1930^{10}$, han servido para complementar los datos demográficos a la hora de elaborar las tablas necesarias para la realización del presente trabajo.

6 F. Contreras Pérez, "Demografía histórica e historia de Género. El caso de la Andalucía urbana”. En III Jornadas Internacionales sobre Historia Contemporánea y nuevas fuentes, Actas. Alicante, Universidad de Alicante, 1999, pp. 113-120.

7 M. J. González Castillejo, La nueva historia. Mujer, vida cotidiana y esfera pública en Málaga (1931-1936). Málaga, Universidad de Málaga, 1991.

8 Archivo Municipal de Huelva (AMH), Legajos 178 y 179. Libros 1, 2, 3, 4 y 5

9 Instituto Geográfico, Catrastal y de Estadística: Censo de la Población de España de 1930, Madrid 1930.

10 Anuario Estadístico de España, 1930. INE. 
Finalmente hemos acudido a una bibliografía ${ }^{11}$, que nos ha permitido situarnos en el contexto y entrar en materia. Las obras seleccionadas comprenden tanto trabajos relativos al periodo contemporáneo onubense como otros de autoras especializadas en estudios de género, entre ellas Mary Nash, María Rosa Capel o Cristina Borderías, que nos han ayudado sobremanera a dibujar el panorama laboral de las españolas de final de los veinte y principios de los treinta.

NOTAS GENERALES ACERCA DEL TRABAJO FEMENINO EN LA ESPAÑA DE LOS AÑOS 30

En el origen del feminismo continental se encuentra la incorporación de la mujer al mundo laboral en las sociedades industrializadas. Sin embargo, no podemos olvidar que la evolución de nuestro país no fue la misma que la europea, debido al fracaso generalizado de la industrialización, a excepción de zonas como Cataluña o el País Vasco.

El trabajo femenino presentaba una serie de peculiaridades que lo hacían diferente al realizado por el varón. Las mujeres se integran en el mercado laboral "por necesidad", ya fuera porque el salario del cabeza de familia no alcanzara a cubrir los gastos domésticos o porque el esposo hubiera fallecido. Aún así, no era cosa aceptada el que las esposas trabajaran, ya que la sociedad entendía que los hijos y el marido quedaban desatendidos. En otro sentido, y en cierta medida, el que la mujer se ocupara fuera de casa podía representar una merma en la autoridad del marido, o la vergüenza de no poder sostener a su familia. Por tanto, el trabajo femenino en líneas generales, no se realiza por vocación sino por condicionantes económicos.

La jornada laboral era la misma para hombres y mujeres, pero la remuneración es notablemente inferior en el caso femenino, lo que se justificaba por diversos factores, entre ellos el de la maternidad. Asimismo, las categorías profesionales de mayor importancia eran ejercidas por el personal masculino, siendo los puestos menores desempeñados por mujeres. Por otra parte, estas mujeres tuvieron que hacer frente a las resistencia de los varones, que veían como el personal femenino iba ocupando parcelas profesionales que antes sólo les correspondían a ellos, sobre todo en periodos de crisis, en los que se las consideraba una "competencia desleal" para los padres de familia ${ }^{12}$. A todo lo anterior hay que añadir que algunas leyes dificultaban el ingreso de las féminas al mundo laboral, a pesar de que la mayoría de ellas nacieron con el fin de proteger el trabajo femenino y la reproducción ${ }^{13}$.

11A priori, contamos con una amplia bibliografía general acerca del periodo que comprende las décadas de los años 20 y 30 en España, pero la información se vuelve bastante más escasa si reducimos el ámbito geográfico a la provincia de Huelva. En cuanto al tema de la mujer, contamos con numerosas obras relativas al ámbito español e incluso andaluz, pero si nos ceñimos al tema del trabajo femenino onubense, no contamos con referencia alguna.

12 C. Borderías, Entre líneas. Trabajo e identidad femenina en la España Contemporánea. La Compañia Telefónica, (1924-1980).Barcelona, Icaria editorial, 1993, p.31.

13 Ibidem, pp. 29-30. 
La segmentación del mundo laboral por género, en lo que se conoce como división sexual del trabajo, es algo típico de estos años. A lo largo del siglo, las mujeres se habían introducido en sectores antaño ocupados por varones, debido a las cualidades específicas de la condición femenina, por ejemplo en el empleo de telefonista, según la división del trabajo por roles en la familia tradicional (servicio doméstico, trabajos de costura, bordados, etc.).

En cuanto al estado civil, solo se veía bien que la mujer casada trabajara en casos de dificultad económica, ya que el hombre tenía como principal función el sustento de la casa.

Las leyes beneficiaban el trabajo de las solteras. Esta legislación se mantuvo vigente hasta la llegada de la República y obligaba a tomar excedencias forzosas por matrimonio, existiendo además el despido por maternidad. Por lo tanto, la mayoría de las mujeres dejaban de trabajar al casarse o como mucho al nacimiento del primer hijo. Sin embargo, durante la República, y contrariamente al supuesto espíritu de igualdad entre sexos que en ella se proclamaba, se limitó aún más el trabajo femenino en algunos sectores ${ }^{14}$.

Sin embargo, la inestabilidad económica de finales de los veinte y comienzos de los treinta hizo que muchas mujeres se incorporaran al mercado del trabajo, lo que supuso un cambio en las relaciones familiares, aunque las tareas del hogar seguían siendo propias de la esposa. En resumidas cuentas, las limitaciones sociales de la época condicionaban totalmente la vida laboral de las mujeres.

La mujer que trabajaba pertenecía generalmente a las clases medias o bajas de la sociedad. En principio, la mujer soltera entregaba su sueldo a los padres, y cuando se casaba lo cedía al marido, pero paulatinamente la mujer fue alcanzando pequeñas cuotas de independencia económica, utilizando parte de su sueldo para algunos gastos personales.

El sector primario acogía a un numeroso grupo de trabajadoras, que ayudaban a sus maridos o padres en el trabajo del campo. En cuanto al sector secundario, las mujeres que trabajaban en la industria percibían salarios inferiores a los varones, a la par que desempeñaban puestos de menor categoría. Pero, es en el sector terciario donde encontramos el mayor número de trabajadoras, ya que es en dicho sector donde se incluyen los oficios vinculados con los roles asignados a la mujer, como el servicio doméstico (criadas o cocineras), o los relacionados con el textil y la costura (modistas, bordadoras o planchadoras). Por otra parte, estos últimos oficios podían ser desarrollados en el propio domicilio, lo que facilitaba la realización de las tareas domésticas. Las mujeres no solían desempeñar a estas alturas del siglo lo que conocemos como profesiones liberales, a excepción de las maestras, comadronas o enfermeras, siendo inusual la existencia de mujeres médicos o abogados, por ejemplo.

14 Ibidem, p.33. 
Para la mujer de aquellos años resultaba muy difícil compaginar su vida familiar con la vida laboral. Los hombres no colaboraban en este tipo de quehaceres, por lo que las señoras debían realizar todas las tareas domésticas (y recordemos que las condiciones técnicas no son las de ahora), además de cumplir con sus deberes profesionales. En otro sentido, la incorporación de la mujer a la vida laboral supuso un importante cambio en las relaciones hombre-mujer, aunque dicho cambio tuviera lugar muy lentamente. La mujer se va a ver ahora relacionada en el lugar de trabajo con personal masculino, algo que no era habitual en una época en que las relaciones entre ambos sexos se limitaban al ámbito familiar.

En definitiva, la vida laboral de la mujer estaba determinada por el acceso al matrimonio. ${ }^{15}$

\section{EL CASO DE HUELVA}

EL CONTEXTO HISTÓRICO

El año 1930 será el punto de partida de una década que tendrá importantes consecuencias en la Historia Contemporánea, tanto española como onubense. Nos situaremos pues, en la dictadura de Dámaso Berenguer, que desde 1930 a 1931, supuso la transición del Régimen de Primo de Rivera a la II República.

Primo de Rivera se vio obligado a nombrar a quien llevara a cabo la transición política hacia una nueva monarquía, recayendo la elección en Dámaso Berenguer, cuyas pretensiones eran restablecer el orden previo a la instauración del Régimen, incluyendo entre otros temas la práctica del caciquismo. De esta manera, el gobierno de la provincia quedaba en manos de las élites económicas, el caciquismo renacía y los concejales conservadores se erigían como mayoría política en el Ayuntamiento ${ }^{16}$. Sin embargo, al igual que sucedió en el resto de la nación española, también en Huelva surgieron grupos de oposición a Berenguer; monárquicos, obreros, estudiantes y republicanos se levantaron en contra. La conocida como "Dictablanda" se iría debilitando ante el continuo acoso de quienes consideraban que la vuelta al pasado no era la solución a los problemas que afectaban al país. Los obreros y estudiantes tomarían las calles, unos para luchar contra las nuevas leyes universitarias, que mermaban los logros de los ańos veinte, otros a causa de las condiciones económicas que España vivía tras el crack del 29.

En nuestra provincia la crisis económica venía afectando ya desde comienzos de la década anterior, a causa de los problemas de la minería, que influyeron por extensión a la industria y al comercio de Huelva. También la pesca se vio afectada por distintos aranceles que la perjudicaron sobremanera. Todas estas dificultades

15 F. Contreras Pérez, "Actores en un escenario urbano: nacer, vivir y morir entre los siglos XIX y XX. En Sindicatos y trabajadores en Sevilla, L. Álvarez Rey y E. Lemus López (coord.). Sevilla, Universidad de Sevilla, 2000, pp. 23-61, p. 58.

16 M. A. García Díaz, "Huelva (1930-1931): Entre la dictadura y la república”. En Huelva en su historia- 2 época, vol. 9, 2002, pp. 123-125. 
atacaron profundamente a la economía onubense, por lo que no se hizo esperar la reacción de los sectores más desfavorecidos contra el nuevo gobierno ${ }^{17}$, pidiendo mayores salarios y mejoras en las condiciones de trabajo, movimientos que se vieron favorecidos por la legalización del sindicalismo tras la caída del la Dictadura.

Es este contexto, de inestabilidad política y problemas económicos, si ya era complicado el acceso al mercado laboral de los varones, cual difícil no lo sería para las mujeres.

\section{EL TRABAJO FEMENINO POR SECTORES EN LA CIUDAD DE HUELVA}

Utilizando el Padrón Municipal de Huelva hemos realizado un muestreo formado por un total de 1785 mujeres. De ellas, únicamente el 9,41 \% realizan algún tipo de trabajo remunerado, porcentaje que aún así, es ligeramente superior a la media española.

TABLA 1: Total numérico y porcentual de la población femenina activa y no activa de Huelva y Espańa en 1930

\begin{tabular}{|l|c|c|c|c|c|}
\cline { 2 - 6 } \multicolumn{1}{c|}{} & TOTAL & \multicolumn{2}{c|}{ NO TRABAJAN } & \multicolumn{2}{c|}{ TRABAJAN } \\
\cline { 2 - 6 } \multicolumn{1}{c|}{} & No & No & $\%$ & No & $\%$ \\
\hline Huelva & 1785 & 1625 & 90,59 & 168 & 9,40 \\
\hline España & 12.111 .989 & 11.007 .994 & 90,90 & 1.103 .995 & 9,10 \\
\hline
\end{tabular}

Fuente: Elaboración propia a través del Padrón Municipal de Huelva de 1930 y de Instituto Geográfico, Catastral y de Estadística: Censo de la Población de España... de 1930, Madrid, 1932.

La próxima tabla representa la distribución de la población activa femenina por sectores. Observamos que el sector terciario es el que acapara un mayor número de trabajadoras, un $70,76 \%$ del total de la población activa femenina, frente al $16,66 \%$ del sector primario y el escaso 3,57\% del sector secundario. Esto indica que las mujeres se empleaban mayoritariamente en el sector servicios, que es el que aglutina los roles femeninos, cuestión que corroboraremos en la siguiente tabla. Por otra parte, debemos mencionar la escasa industrialización existente en esta época en la capital onubense. 
TABLA 2: Distribución de la población activa femenina por sectores

\begin{tabular}{|c|c|c|}
\hline SECTORES & No $^{\circ}$ & $\%$ \\
\hline $1^{\mathrm{o}}$ & 28 & 16,66 \\
\hline $2^{\mathrm{o}}$ & 6 & 3,57 \\
\hline $3^{\mathrm{o}}$ & 134 & 79,76 \\
\hline
\end{tabular}

Fuente: Elaboración propia a través del Padrón Municipal de Huelva de 1930.

La siguiente tabla pone de manifiesto la idea ya comentada anteriormente de que las mujeres se empleaban en oficios típicamente femeninos, como el servicio doméstico, que ocupaba el $42,85 \%$ de las activas, seguidas de modistas y costureras que constituían el 20,23\%.

TABLA 3: Población activa femenina de Huelva en 1930 según actividad laboral

\begin{tabular}{|l|c|c|c|}
\hline \multicolumn{1}{|c|}{ TRABAJO } & CASADAS & SOLTERAS & VIUDAS \\
\hline Sirvientas & 1 & 61 & 10 \\
\hline Costureras & 5 & 18 & 6 \\
\hline Modistas & 0 & 3 & 1 \\
\hline Vendedoras & 1 & 1 & 3 \\
\hline Industriales & 2 & 0 & 3 \\
\hline Cocineras & 0 & 0 & 1 \\
\hline Dependientas & 0 & 1 & 1 \\
\hline Bordadoras & 0 & 1 & 0 \\
\hline Maestras & 2 & 2 & 0 \\
\hline Empleadas & 1 & 0 & 0 \\
\hline Carpinteras & 1 & 0 & 0 \\
\hline Camareras & 0 & 1 & 0 \\
\hline Pupilas & 0 & 3 & 0 \\
\hline Peinadoras & 0 & 2 & 0 \\
\hline Limpiadoras & 1 & 0 & 0 \\
\hline Lavanderas & 1 & 2 & 3 \\
\hline Comestibles & 1 & 1 & 0 \\
\hline Jornaleras & 7 & 13 & 8 \\
\hline Total & 23 & 100 & 35 \\
\hline
\end{tabular}

Fuente: Elaboración propia a través del Padrón Municipal de Huelva de 1930. 
En cuanto al estado civil, eran las solteras quienes mayoritariamente trabajaban fuera de casa, seguidas a distancia de viudas y casadas. Si nos centramos en el servicio doméstico, observamos que son las solteras quienes se dedican generalmente a él, con un $84,72 \%$ del total, mientras que las viudas y casadas suponen el $13,88 \%$ y el $1,38 \%$ respectivamente. De ellas, el grupo de edad predominante se sitúa alrededor de los 25 años, cuando la mujer aún no había contraído matrimonio ${ }^{18}$. Era habitual que cuando las hijas alcanzaban cierta edad, entre los 12 y los 16 ańos, entraran a servir para apoyar la economía familiar, o como forma de reunir la dote para casarse. Estas situaciones ocurrían en la mayoría de los casos en los estratos sociales inferiores.

TABLA 4: Población femenina de Huelva en 1930 según estado civil y laboral

\begin{tabular}{|l|c|c|c|c|c|}
\hline $\begin{array}{c}\text { ESTADO } \\
\text { CIVIL }\end{array}$ & No & Trabajan & $\begin{array}{c}\text { \% que } \\
\text { trabaja }\end{array}$ & No trabajan & \% no trabaja \\
\hline Casadas & 831 & 23 & 2,77 & 808 & 97,23 \\
\hline Solteras & 717 & 109 & 15,20 & 608 & 84,79 \\
\hline Viudas & 235 & 36 & 15,31 & 199 & 84,68 \\
\hline
\end{tabular}

Fuente: Elaboración propia a través del Padrón Municipal de Huelva de 1930.

TABLA 5: Mujeres empleadas en el servicio doméstico en Huelva en 1930, número y porcentaje por estado civil

\begin{tabular}{|l|c|c|}
\hline \multicolumn{1}{|c|}{ ESTADO CIVIL } & No & $\%$ \\
\hline Casadas & 1 & 1,38 \\
\hline Solteras & 61 & 84,72 \\
\hline Viudas & 10 & 13,88 \\
\hline Total & 72 & 100,00 \\
\hline
\end{tabular}

Fuente: Elaboración propia a través del Padrón Municipal de Huelva de 1930.

18 P. Miret Gamundi, Primonupcialidad en España durante el siglo XX: Evolución histórica y comportamientos generacionales. Tesis doctoral. UNED, 2002, p.66. 
TABLA 6: Edad Media de las mujeres empleadas en el servicio doméstico, por estado civil, en Huelva en 1930

\begin{tabular}{|l|c|}
\hline \multicolumn{1}{|c|}{ ESTADO CIVIL } & EDAD MEDIA \\
\hline Casadas & 32 \\
\hline Solteras & 25,09 \\
\hline Viudas & 46,50 \\
\hline
\end{tabular}

Fuente: Elaboración propia a través del Padrón Municipal de Huelva de 1930.

La clase social es también relevante en todos estos datos. Para analizar esta cuestión hemos realizado una división de las empleadas en el servicio doméstico por barrios, observando que es en los de categoría social superior donde se emplean un mayor número de domésticas. Destacamos así, La Merced, La concepción, Colón y San Pedro respectivamente ${ }^{19}$. Las criadas aparecían a menudo inscritas en la misma casa donde trabajaban, ya que la mayoría de ellas lo hacían en régimen de internas.

TABLA 7: No de empleadas en el servicio doméstico por barrios

\begin{tabular}{|l|c|}
\hline \multicolumn{1}{|c|}{ BARRIOS } & No \\
\hline La Merced & 20 \\
\hline La Concepción & 10 \\
\hline S. Pedro y S. Fco. & 9 \\
\hline Colón & 24 \\
\hline S. Sebastián & 1 \\
\hline Reina Victoria y Paseo Sur & 1 \\
\hline Molino & - \\
\hline La Cinta & - \\
\hline Carretera de Sevilla & 2 \\
\hline
\end{tabular}

Fuente: Elaboración propia a través del Padrón Municipal de Huelva de 1930.

La edad media al matrimonio es un dato muy interesante a la hora de analizar el trabajo femenino, estando íntimamente relacionado con éste, si tenemos en cuenta que la mayoría de las trabajadoras remuneradas eran solteras.

19 A. Díaz Zamorano, Huelva. La construcción de una ciudad. Huelva, Ayuntamiento de Huelva, 1999, p. 300. 
TABLA 8: Indicadores de Nupcialidad: Celibato definitivo y Edad media al matrimonio (SMAM) para hombres y mujeres en 1930

\begin{tabular}{|l|c|c|c|c|}
\hline CIUDADES & $\begin{array}{c}\text { Celibato Def } \\
(\mathrm{v})\end{array}$ & $\begin{array}{c}\text { Celibato Def } \\
(\mathrm{m})\end{array}$ & $\begin{array}{c}\text { SMAM } \\
(\mathrm{v})\end{array}$ & $\begin{array}{c}\text { SMAM } \\
(\mathrm{m})\end{array}$ \\
\hline Huelva & 7,86 & 8,87 & 28,90 & 27,28 \\
\hline Provincia de Huelva & 4,23 & 4,08 & & \\
\hline & & & & \\
\hline Almería & 6,77 & 10,11 & 26,85 & 24,98 \\
\hline Cádiz & 14,85 & 18,36 & 29,52 & 26,40 \\
\hline Córdoba & 10,15 & 12,98 & 29,01 & 26,11 \\
\hline Granada & 8,96 & 12,16 & 27,87 & 26,34 \\
\hline Jaén & 7,95 & 10,33 & 28,58 & 24,86 \\
\hline Málaga & 7,55 & 11,81 & 28,56 & 26,72 \\
\hline Sevilla & 12,97 & 16,83 & 29,64 & 27,83 \\
\hline & & & & \\
\hline España & 7,64 & 11,60 & 27,91 & 25,9 \\
\hline Andalucía urbana & 9,63 & 12,68 & 28,49 & 26,32 \\
\hline
\end{tabular}

Fuentes: El Celibato Definitivo (Celibato Def) se ha hallado a través de Instituto Geográfico, Catastral y de Estadística: Censo de la Población de España... de 1930. Madrid, 1932; y para la Edad Media al Matrimonio (SMAM) hemos utilizado Contreras Pérez, F.: "Demografía histórica e historia de Género. El caso de la Andalucía Urbana", en III Jornadas Internacionales sobre Historia Contemporánea y nuevas fuentes. Alicante, Universidad de Alicante, 1999; y Miret Gamundi, P.: Primonupcialidad en España durante el siglo XX: Evolución histórica y comportamientos generacionales. Tesis doctoral. Barcelona,Universidad Nacional de Educación a Distancia, 2002. Datos obtenidos sobre el total del Censo.

En las primeras décadas del siglo XX tuvo lugar una importante caída en los matrimonios, descenso que continuó hasta finalizar la década de los treinta. ${ }^{20}$

Si analizamos la tabla anterior, vemos que las mujeres llegaban al primer matrimonio a una edad inferior a la del hombre. Las motivaciones económicas son la principal causa de este fenómeno, de manera que el varón necesita de una estabilidad económica antes de contraer nupcias, ya que será el encargado de mantener a la familia. Por el contrario, la mujer en aquella época está exenta de ese deber, por lo que contraerá matrimonio a una edad inferior. ${ }^{21}$. Muchas mujeres solteras, sobre todo pertenecientes a las clases sociales medias y bajas, se dedican

20 Ibidem, p. 42.

21 M. G. Nuñez Pérez, Trabajadoras en la Segunda República. Un estudio sobre la actividad económica extradoméstica (1931-1936). Madrid, Ministerio de Trabajo y Seguridad Social, 1989, pp.67-68. 
al trabajo remunerado con el fin de reunir la dote o para ayudar a su familia, pero normalmente lo abandonan cuando contraen matrimonio. Si nos centramos en la capital de Huelva, vemos que la edad media al matrimonio es notablemente superior, 27,3 frente a la media española 25,9, es decir, las onubenses se casaban a una edad superior que el resto de las espańolas. Posiblemente la coyuntura económica de Huelva, que era francamente desfavorable desde unos años atrás, influyera en este dato, haciendo más dificultoso obtener los medios económicos para mantener una familia. De esta forma, tanto hombres como mujeres se veían obligados a matrimoniar más tardíamente.

\section{LA PROSTITUCION EN LA HUELVA DE I930}

El ejercicio de la prostitución ha estado tradicionalmente ligado a la mujer, siendo un tema que ha preocupado a la sociedad en todas las épocas históricas. Aunque en los últimos años han aumentado los estudios acerca del que se denomina "el oficio más viejo del mundo", relativos a la provincia onubense apenas existen trabajos de este tipo, si exceptuamos un breve apunte publicado en 1990, referido a una casa de prostitución situada en la localidad de Zalamea la Real, a cargo de Sebastián Carrasco ${ }^{22}$, algunas notas que Andrés Moreno Mengíbar y Francisco Vázquez García presentan en su obra Historia de la prostitución en Andalucia ${ }^{23}$, algunas referencias que aparecen en el trabajo de Lucía Prieto Borrego ${ }^{24}$ y unos breves apuntes que recoge $\mathrm{M}^{\mathrm{a}}$ Antonia Peńa ${ }^{25}$ estando, por otra parte, este tipo de estudios muy ligados al aspecto de la marginación, la moralidad o el higienismo, nunca desde la perspectiva del mundo laboral. Es por ello, que creemos interesante elaborar un estudio acerca de este colectivo, con el fin de otorgar voz histórica a un grupo de mujeres trabajadoras que invariablemente han permanecido marginadas por la sociedad. Al igual que la investigación acerca del trabajo remunerado de la mujer onubense en 1930 formará parte de un estudio mucho más ambicioso, el análisis de la prostitución en la Huelva de aquel año, supone el inicio de un proyecto que abarcará ese tema hasta la década de los 50 del siglo XX.

El estudio histórico de este tema se hace complejo debido a la problemática de las fuentes, que generalmente se limitan a regulaciones municipales acerca de la regulación de las casas de citas, normativas higienistas o tratados médicos.

22 S. Carrasco Perea, "Las pupilas", en Revista de Feria de Zalamea la Real, Zalamea la Real, vol. II, núm. 12, 3a época, 1990, pp. 15-17.

23 A. Moreno Mengíbar y F. Vázquez García, Historia de la prostitución en Andalucía. Sevilla, Fundación José Manuel Lara, 2004.

24 L. Prieto Borrego, "La prostitución en Andalucía durante el primer franquismo". En Baética: Estudios de arte, geografía e historia, № 28, 2, 2006 , págs. 665-688.

25 M. A. Peña Guerrero, El tiempo y las fuentes de su memoria. Historia Moderna y Contemporánea de la provincia de Huelva. Tomo IV. La provincia de Huela en los siglos XIX y XX. San Juan del puerto, 1995. 
A la altura de 1930, la prostitución fue, como en la mayoría de los tiempos, un tema tabú para la sociedad, necesidad para unos, vicio para muchos. Sin embargo, el comercio sexual era en aquella época una actividad tolerada y reglamentada ${ }^{26}$, básicamente con la finalidad de evitar la propagación de enfermedades venéreas y los escándalos públicos que el oficio pudiera desencadenar. La mencionada reglamentación obligaba a la inscripción de las prostitutas en un registro y los pertinentes exámenes médicos periódicos. Por tanto, no debemos hablar de tolerancia hacia la profesión de meretriz, sino más bien de un control del oficio. Numerosos grupos hacían oír sus voces en contra de esta "tolerancia", grupos entre los que encontramos a feministas, médicos, políticos, etc., hasta que finalmente se aprobó la legislación de 1935, que acabaría con la normalización del comercio sexual, pasando a ser considerado un delito.

Varios son los factores que han empujado a lo largo del tiempo a la mujer al ejercicio de la prostitución. En el primer tercio del siglo XX, estos factores estuvieron generalmente relacionados con las necesidades económicas y la ausencia de cultura. Las duras condiciones económicas de finales de los veinte y comienzos de los treinta a menudo empujaron a las mujeres a buscar en la ciudad nuevas formas de ganarse la vida y en ocasiones se vieron obligadas a practicar actividades relacionadas con el comercio carnal. Sabemos incluso que existen casos de prostitución encubierta, a menudo a manos de las propias madres para poder subsistir, o con la finalidad de mantener u obtener un puesto de trabajo ${ }^{27}$.

A través del Padrón de 1930 hemos podido realizar un pequeño esbozo del perfil sociohistórico de esta actividad en Huelva. Evidentemente no podemos deducir de los datos estadísticos las motivaciones que empujaron a estas mujeres a dedicarse a este tipo de trabajo, pero sí podemos acercarnos un poco más a este segmento de población femenina.

Como en el resto del país, en Huelva la prostitución era una actividad regularizada a la altura de 1930, la ciudad contaba con dichos reglamentos ya en $1883^{28}$. En el Padrón Municipal de Huelva hemos podido observar cómo se ejerció este tipo de actividad en los burdeles, casas dirigidas por una "pupilera", cuya profesión aparece registrada como "sus labores", pero que se dedica a la explotación de las prostitutas, las pupilas, con las que convive en la misma vivienda, acompañadas de alguna sirvienta.

26 Desde mediados del siglo XIX se fue instaurando en todo el territorio español una política de tolerancia reglamentada de la prostitución, a causa de la visión del tema como un problema social, consecuencia de la modernización económica de la época. Ver A. Moreno Mengíbar y F. Vázquez García, Historia de la prostitución..... pp.147-148.

27 M. R. Capel Martínez, "La prostitución en España: Notas para un estudio socio-histórico". En Mujer y Sociedad en España (1700-1975). Madrid, 1982, p.272.

28 J.L. Guereña, La prostitución en la España contemporánea. Madrid, Marcial Pons, 2003, p.278. 
La Reglamentación exigía a los burdeles que cumplieran ciertos requisitos de orden moral. No podían estar situados en torno a espacios comerciales y a los lugares de tránsito de las gentes respetables ${ }^{29}$, por lo que era habitual que determinadas zonas albergaran la mayoría de los lupanares de la ciudad, aunque no podemos decir que existiera un emplazamiento fijo para estos negocios.

En Huelva han sido las calles Gran Capitán o Diputación, lugares tradicionales de ejercicio de la prostitución, hasta no hace mucho tiempo ${ }^{30}$. A través del Padrón, observamos notables peculiaridades de estas calles, que dan fe del uso de la mayoría de las viviendas sitas en ellas. Observamos que los hogares registrados no corresponden al típico núcleo familiar de la época. De los 28 hogares inscritos, aparecen tan solo dos matrimonios con hijos, un viudo que vive solo, varios hogares cuyos cabezas de familia son mujeres, tres casas de huéspedes, seis prostíbulos abiertamente registrados y una seńorita dedicada en solitario al oficio.

En cuanto a los hogares cuyas cabezas de familia son mujeres, encontramos el caso de una mujer de 27 años que convive con un varón de 25, que no aparece registrado como familiar o huésped, y una criada. Otro ejemplo es el de una mujer sola, de 45 ańos, que vive con un "pariente" varón de 40. También vemos varios casos de madres solteras, situación que en aquella época era muy dura y que podía ser tanto causa como consecuencia del ejercicio de la prostitución. En una ocasión, la mujer declara trabajar de criada, en otra, la madre soltera convive con su hija, un huésped y una criada. Quizás esto último suponga un caso de prostitución encubierta, podría ser una casa de prostitución disfrazada de pensión, o tal vez, una mujer que se ve obligada a alquilar una habitación para sostener a su hija, aunque esto último no se corresponde con la existencia de la criada.

Por lo que se refiere a las tres casas de huéspedes, es posible, aunque no podamos probarlo, que señalen relaciones de prostitución más o menos encubiertas. No olvidemos que no siempre se admitía a las claras el ejercicio del oficio, por lo que a menudo existían casas de huéspedes que acogían a parejas formadas por una prostituta y su cliente para pasar unas horas o la noche. En las diferentes ciudades andaluzas, estos lugares recibían distintos nombres ${ }^{31}$, y aunque no sabemos cómo se denominaban en nuestra ciudad, no sería descabellado suponer que más de una se disfrazaban de pensiones y casas de huéspedes.

Respecto a los seis prostíbulos, no encontramos censados hombres en estas casas, lo que hace suponer que son las mujeres, la mayoría de ellas de mediana edad y solteras, quienes controlaban este tipo de negocios. Estas señoras aparecen registradas como "cabeza de familia" y como ocupación declaran "su casa". Junto

29 F. Vázquez García y A. Moreno Mengíbar, Historia de la prostitución...., p. 170.

30 Ma Antonia Peńa describe en El tiempo y la fuentes....., p.184, como ya en 1908 la Junta Municipal de Reformas Sociales de Huelva recomendaba el traslado de los burdeles a la calle Gran Capitán y a la carretera de Gibraleón.

31 F. Vázquez García y A. Moreno Mengíbar, Historia de la prostitución...., p.172. 
a ellas, en la misma vivienda se encuentran empadronadas varias mujeres jóvenes que hacen constar como profesión la de "pupila". ${ }^{32}$ La reglamentación del oficio hacía necesario la jerarquización del burdel, en la que el ama ejercía el control de sus pupilas ${ }^{33}$, por ello aparece siempre registrada como "cabeza de familia". En ningún caso hemos encontrado de profesión "ama” o "pupilera”, como se las denominaba en la época.

No siempre quienes se dedicaban a esta actividad lo hacían bajo el "amparo" de un ama, en ocasiones la prostitución se ejercía por libre, eran las denominadas carreristas, que podemos identificarlas en el Padrón porque declaran ser cabezas de familia y de profesión "pupila". Tenían quizás una mayor libertad de actuación, ya que vivían en su propio domicilio y no bajo el control de la pupilera, que generalmente ejercía una férrea disciplina sobre las jóvenes.

La mayoría de las mujeres dedicadas a estas labores eran solteras, aunque se dan varios casos de casadas, concretamente tres, aunque en dichas viviendas no aparece registrado marido alguno. Si exceptuamos un caso, que se declara nacida en Huelva, las otras dos provienen de otras ciudades, como Cádiz y Ayamonte, por lo que es de presumir que la mujer se traslada de su ciudad de origen dejando allí al esposo.

TABLA 9: Estado civil de las mujeres (pupilas) ${ }^{34}$ dedicadas a la prostitución en Huelva en 1930

\begin{tabular}{|c|c|c|}
\hline ESTADO CIVIL & No & $\%$ \\
\hline Casadas & 3 & 13,04 \\
\hline Solteras & 20 & 86,95 \\
\hline Viudas & 0 & 0 \\
\hline Total & 23 & 100 \\
\hline
\end{tabular}

Fuente: Elaboración propia a través del Padrón Municipal de Huelva de 1930

En cuanto al nivel de educación encontramos que, de las siete mujeres que se suponen que son amas, es decir, quienes aparecen inscritas como cabezas de familia, cinco no saben leer ni escribir, lo que supone un 71,42\% del total. Parecidos son los datos referentes a las pupilas, ya que un 78,26\% de las mismas son analfabetas.

32 Este tipo de hogares suponen 5 del total de 9 contabilizados, es decir un 55,55 \% del total del muestreo.

33 F. Vázquez García y A. Moreno Mengíbar, Historia de la prostitución..., p.172.

34 No se computan las pupileras. 
TABLA 10: Procedencia de estas mujeres

\begin{tabular}{|l|c|c|}
\hline \multicolumn{1}{|c|}{$\begin{array}{c}\text { LUGAR DE } \\
\text { ORIGEN }\end{array}$} & No & $\%$ \\
\hline Huelva & 4 & 17,39 \\
\hline Provincia de Huelva & 6 & 26,08 \\
\hline Fuera de la provincia & 13 & 56,52 \\
\hline Total & 23 & 100 \\
\hline
\end{tabular}

Fuente: Elaboración propia a través del Padrón Municipal de Huelva de 1930

TABLA 11: Nivel de albabetización de las pupilas en Huelva en 1930

\begin{tabular}{|l|c|c|l|c|c|}
\hline $\begin{array}{c}\text { Saben } \\
\text { leer }\end{array}$ & No & $\%$ & $\begin{array}{c}\text { Saben } \\
\text { escribir }\end{array}$ & No & $\%$ \\
\hline $\mathrm{Si}$ & 5 & 21,73 & $\mathrm{Si}$ & 5 & 21,73 \\
\hline $\mathrm{No}$ & 18 & 78,26 & No & 18 & 78,26 \\
\hline Total & 23 & 100 & Total & 23 & 100 \\
\hline
\end{tabular}

Fuente: Elaboración propia a través del Padrón Municipal de Huelva de 1930

El Padrón Municipal de Huelva nos presenta pequeños esbozos de la vida de estas mujeres, a las que también queremos considerar trabajadoras remuneradas.

Sabemos, por ejemplo, que en el no 5 de la calle Gran Capitán, Doña Julia, soltera de 53 años de edad, y registrada como cabeza de familia, regentaba un burdel en el que tenía a su cargo cuatro pupilas, de edades comprendidas entre 28 y 35 años, todas solteras. Dos de ellas, Alicia, de 30 años de edad y Virginia, de 28 eran portuguesas; María procedía de la provincia de Sevilla y sólo una, Ángeles, era onubense.

Pero, no es este el prostíbulo más importante, por lo menos en lo que se refiere al número de pupilas. En el no 17 de la misma calle hallamos el burdel regentado por Eva, soltera, de la que desconocemos su edad, pero que como profesión refiere su casa. Siete chicas entre los 27 y 34 años conviven con ella: Aurora Ma, portuguesa; Manuela, jienense; Rosario, onubense; María, sevillana; Estrella, natural de Isla Cristina y Josefa y Agustina, ayamontinas. Junto a ellas vive Felisa, de 50 ańos, que aparece como criada y que procede de Fregenal. Podríamos decir que estamos ante el burdel típico de aquella época, por lo menos en Huelva. Un número modesto de chicas, que indica que estos lugares no eran muy amplios, posiblemente por ser Huelva una ciudad pequeña. Las mujeres no eran demasiado jóvenes, no estamos hablando de adolescentes, y la mayoría provenían 
de otros lugares. Hemos encontrado varias portuguesas entre las prostitutas, así como algunas chicas procedentes de los pueblos de la provincia y de Sevilla. A veces estas mujeres dejaban su lugar de origen debido a situaciones de pobreza $\mathrm{u}$ otras cuestiones de tipo personal, en muchas ocasiones y ante las dificultades halladas, la prostitución era la única alternativa posible.

Asimismo, aparecen en el Padrón amas con un número pequeño de pupilas. Por ejemplo, las tres mujeres que trabajaban en el no 6 de Gran Capitán, de 25 años, bajo el mando de María, de 28 (apreciamos aquí un ama de menor edad a lo habitual, o quizás que las cuatro mujeres ejercieran juntas y una de ellas, la mayor, se declarara como cabeza de familia). Lo mismo parece ocurrir en el no 11 , donde Fani y Soledad de 37 y 32 años conviven juntas. En este caso es la segunda la que aparece como cabeza de familia, pero ambas se inscriben como pupilas.

Estas y otros indicios de una realidad semiclandestina se entresacan del Padrón, haciendo que nos movamos en la mayoría de los casos en el terreno de la conjetura. Sin embargo, esta fuente nos proporciona algunos datos que de otra forma sería difícil de obtener, ya que en los Censos de Población aparecen estas mujeres en el apartado de vagabundos y desempleados. En el Padrón, sin embargo, aparece su profesión como eso, una profesión, lo que no quiere decir que esta fuera una ocupación aceptada por la sociedad, sino más bien controlada por las autoridades.

Casos parecidos encontramos en las calles Diputación y Granada, de la misma zona. Concluimos, por tanto, que quienes viven allí no forman los típicos hogares, sino que constituyen un grupo especial de la población onubense de 1930.

\section{CONSIDERACIONES FINALES}

A la luz de las fuentes utilizadas hemos encontrado que el papel de la mujer onubense, en la fecha que nos ocupa, queda prácticamente relegado al ámbito del hogar, al igual que ocurre en casi todo el país, si exceptuamos zonas donde el trabajo femenino tenía una mayor representación en el mundo laboral, como pudieran ser Cataluña o Navarra. Esta situación estuvo en gran medida causada por la profunda crisis económica padecida por nuestra provincia en la década de los veinte y principios de los treinta, pero también por el tipo de sociedad patriarcal predominante en la época.

De las escasas mujeres que salen de casa para trabajar, la mayoría eran solteras. La mujer casada generalmente realizaba trabajos extradomésticos en casos de necesidad económica, ya que estaba mal considerado que una mujer casada trabajara, debido a que correspondía al esposo esa responsabilidad. Tras las solteras, eran las viudas quienes en segundo lugar se incorporaban a la esfera laboral, de igual manera a causa de los aprietos económicos. Aun así existen excepciones como es el caso de las dos médicas colegiadas en Huelva en $1930^{35}$, que presuponemos no

35 T. Ortiz Gómez, "La mujer como profesional de la medicina en la España contemporánea: El caso de Andalucía (1898-1981”). En Dynamis, n 5-6, 1985-86, pp. 343-366. 
ejercían por necesidad. A pesar de ello, consideramos que la mujer no trabajaba por el hecho de realizare personalmente, ya que aún esa cultura no estaba muy extendida.

El trabajo de la mujer estaba relegado a unas parcelas concretas, generalmente relacionadas con los roles femeninos, es decir, las onubenses trabajaban en oficios vinculados a las tareas del hogar, habitualmente en el servicio doméstico o la costura. Excepciones a la regla eran quienes se dedicaban al comercio, las maestras o las prostitutas, a las que hemos dedicado un epígrafe en nuestro trabajo.

En cuanto a este gremio, creemos que la prostitución era una actividad reglamentada, lo que se refleja en el hecho de que en el Padrón Municipal aparezca como una profesión más. Las meretrices aparecían empadronadas, y en la casilla que determinaba la ocupación encontramos el calificativo de "pupila", para referirse a ellas.

Aún así, en ocasiones los burdeles se intentan disfrazar de casas de hospedaje, lo que nos indica en cierta medida la percepción moral que la sociedad tenía de este colectivo. 
